\title{
AUTOCLINISMS AND AUTOMORPHISMS OF FINITE GROUPS II
}

\author{
by JÜRGEN TAPPE
}

(Received 8 July, 1979)

In part I of this paper P. Hall's formula for finite stem groups was derived. Using results of C. R. Leedham-Green and S. McKay, a similar formula for isoclinic groups with arbitrary branch factor group is shown.

The main result of this paper is the following theorem, which appears without proof in $[1$, p. 203].

THEOREM. Let $\Gamma$ be an isoclinism family of finite groups, $Q$ a finite abelian group and $\mathrm{Acl}(\Gamma)$ the autoclinism group of $\Gamma$. Then we have

$$
\frac{1}{|\operatorname{Acl}(\Gamma) \times \operatorname{Aut}(Q)|}=\sum \frac{1}{|\operatorname{Aut}(G)|},
$$

where $G$ runs through a complete system of non-isomorphic groups in $\Gamma$ with $Q$ as branch factor group.

Let $G$ and $H$ be groups (not necessarily finite). We call $G$ and $H$ strongly isoclinic, if there exists an isomorphism $\alpha$ from $G /\left(Z(G) \cap G^{\prime}\right)$ to $H /\left(Z(H) \cap H^{\prime}\right)$, which induces an isomorphism $\gamma$ from $G^{\prime}$ to $H^{\prime} ; \alpha$ is called a strong isoclinism, if $\mathrm{G}=\mathrm{H}$ a strong autoclinism. It can be easily verified that a strong isoclinism induces isomorphisms $\alpha_{1}$ from $G / Z(G)$ to $H / Z(H)$ and $\alpha_{2}$ from $G_{a b}$ to $H_{a b}$, where $\alpha_{1}$ and $\alpha_{2}$ "coincide" on $G /\left(G^{\prime} Z(G)\right)$, and which determine $\alpha$. The pair $\left(\alpha_{1}, \gamma\right)$ is an "ordinary" isoclinism from $G$ to $H$. The restriction of $\alpha_{2}$ to $\left(G^{\prime} Z(G)\right) / G^{\prime}$ is an isomorphism onto $\left(H^{\prime} Z(H)\right) / H^{\prime}$. These quotients are called the branch factor groups of $G$ and $H$, being invariant under strong isoclinism. In the terminology of $\mathbf{P}$. Hall, strong isoclinism describes the "situation of the commutator quotients".

Let $\alpha$ be a strong autoclinism of $G, K=G / Z(G), Q$ the branch factor group of $G$, and $\tau$ the restriction of $\alpha_{2}$ to $Q$. Then $\alpha$ determines an element $\left(\left(\alpha_{1}, \gamma\right), \tau\right)$ of $\operatorname{Acl}(\Gamma) \times$ $\operatorname{Aut}(Q)$. Let $\Phi$ denote the class of groups being strongly isoclinic to $G$, and $A(\Phi)$ the corresponding group of strong autoclinisms (which does not depend on the representatives of $\Phi)$. Then we have a homomorphism from $A(\Phi)$ to $\operatorname{Acl}(\Gamma) \times \operatorname{Aut}(Q)$, and it is easy to see that the kernel of this homomorphism is isomorphic to $\operatorname{Hom}\left(K_{a b}, Q\right)$. Let $L=$ $G /\left(Z(G) \cap G^{\prime}\right)$ and $B=Z(G) \cap G^{\prime}$, and we consider the central extension

$$
\mathrm{C}: 1 \rightarrow \mathrm{B} \rightarrow \mathrm{G} \rightarrow \mathrm{L} \rightarrow 1
$$

Then $C$ determines an epimorphism from the Schur multiplier of $L$ onto $B$, which corresponds to a coset $\Omega$ of $\operatorname{Ext}\left(L_{a b}, B\right)$, regarded as a subgroup of $H^{2}(L, B)$, (in part I the

Glasgow Math. J. 21 (1980) 205-207. 
Ext-group was denoted by $\overline{\mathrm{sym}}$ ). It follows from Theorem I.2.1 in [2] and similar observations as in part $I$ that the isomorphism classes of groups in $\Phi$ correspond to the orbits of $A(\Phi)$ on $\Omega$, where $\alpha \in A(\Phi)$ acts on $H^{2}(L, B)$, resp. $\Omega$ via the action of $\alpha$ on $L$ and $\gamma$ on $B$. For finite groups we obtain in the same way as in part $I$ the formula

$$
\frac{1}{|A(\Phi)|}=\sum \frac{1}{|\operatorname{Aut}(H)|}
$$

where $H$ runs through the isomorphism classes of groups in $\Phi$.

Now we consider all groups in a family $\Gamma$ with a fixed branch factor group $Q$, which are divided into certain classes $\Phi$ of strongly isoclinic groups. Let $S$ be a (fixed) stem group in $\Gamma$ with $K=S / Z(S)$. We consider all abelian extensions $D$ of $Q$ by $K_{a b}$ :

$$
D: 1 \rightarrow Q \rightarrow \bar{D} \rightarrow K_{a b} \rightarrow 1,
$$

and denote for each $D$ by $G(D)$ the direct product of $S$ and $\vec{D}$ with amalgamated quotient $K_{a b}$. From Theorem II.3.2 in [2] we obtain that each group in $\Gamma$ with $Q$ as branch factor group is strongly isoclinic to some $G(D)$. In order to determine the isomorphism classes, we only have to decide, which groups $G(D)$ are in the same class $\Phi$. The groups $G(D)$ are in one-to-one correspondence with the elements of $\operatorname{Ext}\left(K_{a b}, Q\right)$. As each autoclinism of $S$ induces an automorphism of $K_{a b}$, we have an action of $\operatorname{Acl}(\Gamma) \times \operatorname{Aut}(Q)$ on $\operatorname{Ext}\left(K_{a b}, Q\right)$, and it is not very difficult to see that groups of the form $G(D)$ are strongly isoclinic, if and only if the corresponding elements of $\operatorname{Ext}\left(K_{a b}, Q\right)$ are conjugate under $\operatorname{Acl}(\Gamma) \times \operatorname{Aut}(Q)$. The corresponding stabilizers are the homomorphic images of the groups $A(\Phi)$. For finite groups we obtain

$$
\left|\operatorname{Ext}\left(K_{a b}, Q\right)\right|=\sum \frac{|\operatorname{Acl}(\Gamma) \times \operatorname{Aut}(Q)|}{|A(\Phi)| /\left|\operatorname{Hom}\left(K_{a b}, Q\right)\right|},
$$

where the sum is taken over all classes $\Phi$ of strongly isoclinic groups in $\Gamma$ with $Q$ as branch factor group, which yields

$$
\frac{1}{|\operatorname{Acl}(\Gamma) \times \operatorname{Aut}(Q)|}=\sum \frac{1}{|A(\Phi)|} .
$$

Now the theorem follows from (1) and (2).

Remarks. The theorem above can also be obtained by a dual procedure, using Hall's "situation of the centrals". It is also possible to "extend" the formulae and the results on the isomorphism classes of groups in a family to isoclinism classes of arbitrary central extensions without any further complications.

\section{REFERENCES}

1. P. Hall, On groups of automorphisms, J. Reine Angew. Math. 182 (1940), 194-204.

2. C. R. Leedham-Green and S. McKay, Baer invariants, isologism, varietal laws and homology, Acta Math. 137 (1976), 99-150. 
3. R. Reimers and J. Tappe, Autoclinisms and automorphisms of finite groups, Bull. Austral. Math. Soc. 13 (1975), 439-449.

LeHRSTUHL B FÜr MATHEMATIK

Rheinisch-Westfälische Technische Hochschule, AACHEN,

GERMANY 